Repensando as conexões entre a formação na universidade e as experiências de campo na formação de professores em faculdades e universidades*

Ken Zeichner**

\title{
Resumo
}

Neste artigo, discuto um dos problemas centrais que tem afligido, já há alguns anos, os cursos de formação inicial de professores nas faculdades e nas universidades, a desconexão entre os componentes curriculares acadêmicos e a parcela da formação docente que acontece nas escolas. Primeiro, extrairei de minha experiência como formador de professores e administrador durante mais de trinta anos na Universidade de Wisconsin - Madison e da literatura, elementos para discorrer sobre as várias dimensões dessa questão. Assim, usando o conceito de hibridismo e "terceiro espaço", discutirei vários trabalhos, em andamento em programas formativos nos Estados Unidos, promissores quanto à qualificação da aprendizagem docente nos cursos de formação de professores das universidades e das faculdades, assim como a habilidade dos graduados dos cursos de formação de professores para realizar práticas de ensino desejadas em espaços escolares complexos. Esse trabalho de criação de espaços híbridos na formação de professores no qual o conhecimento empírico e acadêmico e o conhecimento que existe nas comunidades estão juntos de modos menos hierárquicos a serviço da aprendizagem docente representam uma mudança de paradigma na epistemologia dos programas de formação de professores. Discuto que essa mudança rumo a modos mais democráticos e inclusivos de trabaIhar com escolas e comunidades é necessária para as faculdades e as universidades, a fim de que elas possam cumprir sua missão na formação de professores.

Palavras-chave: Formação de professores; Universidade-escola; Programas formativos.

\section{Rethinking the connections between campus courses and field experiences in College and University-based teacher education}

\begin{abstract}
In this paper, I discuss one of the central problems that has plagued college and university-based pre-service teacher education for many years, the disconnect between the campus and school-based components of programs. First, I will

\footnotetext{
* Tradução concedida realizada por Marcelo de Andrade Pereira (UFSM) e Revisão técnica: Gilberto Icle (UFRGS) e Hamilton de Godoy Wielewicki (UFSM).

** Boeing Professor of Teacher Education - University of Washington-Seattle.
} 
draw on my own experiences as a teacher educator and administrator over the last thirty plus years at the University of Wisconsin-Madison and the literature to lay out various dimensions of this issue. Then, utililizing the concept of hybridity and "third space," I will discuss a variety of current work in programs across the U.S. that offers much promise in deepening the quality of teacher learning in college and university-based teacher education programs and the ability of teacher education graduates to enact desired teaching practices in complex school settings. This work in creating hybrid spaces in teacher education where academic and practitioner knowledge and knowledge that exists in communities come together in new less hierarchical ways in the service of teacher learning represents a paradigm shift in the epistemology of teacher education programs. I argue that this shift toward more democratic and inclusive ways of working with schools and communities is necessary for colleges and universities to fulfill their mission in the education of teachers.

Keywords: Education of teachers; Campus and school-based; Programs across.

\begin{abstract}
Mantidos com estudantes de pós-graduação, professores horistas e temporários e com poucos recursos para fazer a colocação de estagiários em campo, os programas de certificação do professor nos Estados Unidos são tidos como as Cinderelas da universidade norte americana. Ideias e dinheiro raramente são gastos na coordenação entre aquilo que é aprendido na academia com o que acontece nas escolas (FEATHERSTONE, 2007, p. 210).

Frequentemente, a parcela de experiência prática da formação de professores tem sido deveras descuidada, dependendo das idiossincrasias de uma colocação pouco criteriosa de estagiários, com pouca orientação acerca do que nela acontece e com pouca conexão com o trabalho feito na universidade (DARLING-HAMMOND, 2009, p. 11).
\end{abstract}

Neste artigo, para que eu possa centrar-me nas conexões entre campo de trabalho e universidade, utilizo o termo conhecimento acadêmico para representar as diversas formas de conhecimento e de especialidade que existem em meio ao corpo docente e técnico nas faculdades e nas universidades. Ao adotá-lo, admito que isso implique uma sobre-simplificação e que existam várias culturas dentro das faculdades e das universidades que, frequentemente, se encontram em tensão entre elas, dentro e fora das escolas, faculdades e departamentos de educação (BULLOUGH et al. 1997; GOODLAD, 1990; LABAREE, 2004). O uso que faço do termo conhecimento acadêmico inclui tanto os conhecimentos adquiridos nas disciplinas de humanidades e ciências, quanto aqueles na área da educação. Um exame das tensões internas nas instituições de formação de professores vai além do escopo deste trabalho. 
Repensando as conexões entre a formação na universidade e as experiências de campo na formação de professores em faculdades e universidades

Durante a maior parte de minha carreira como professor universitário, formador de professores, fui responsável pela organização e acompanhamento das experiências de campo em escolas e comunidades de professores em formação, assim como pela pesquisa sobre os processos de aprendizagem docente nos programas de formação inicial de professores. Um dos desafios mais difíceis, para mim, durante esses anos, tem sido o de mobilizar energia intelectual em meu departamento para reforçar as conexões entre o que os nossos estudantes-professores fazem nos seus estágios em escolas e comunidades e o restante da sua formação docente. Na maior parte dos casos, a supervisão do trabalho desses estudantes nas escolas e as disciplinas ministradas na universidade têm sido feitas, ao menos na University of Wisconsin-Madison (UWMadison), por doutorandos e este trabalho serve como sua fonte principal de recursos durante seu período de pós-graduação (ZEICHNER, 2005).

Ainda que a maioria desses estudantes de pós-graduação esteja interessada em realizar um trabalho de excelência no ensino e/ou na supervisão de professores em formação, muitos deles não estão interessados na formação de professores como um campo de estudo e não participam de quaisquer disciplinas de pós-graduação disponíveis para eles e que digam respeito à formação de professores e à aprendizagem do ensinar. $\mathrm{E}$ mesmo que esses sejam especialistas no ensino ou em alfabetização ou em matemática tenham um considerável número de anos bem sucedidos de ensino na Educação Básica, isso não quer dizer que eles estejam a par do que se sabe por intermédio das pesquisas, sobre como dar apoio à aprendizagem do professor e transferi-la para os primeiros anos do ensino no contexto dos programas de formação de professores nas universidades (por exemplo, Cochran-Smith \& Zeichner, 2005; Darling-Hammond, 2006; Smagorinsky, Cook \& Johnson, 2003), assim como não se pensam como formadores de professores.

Mesmo quando os estudantes de pós-graduação têm o conhecimento e a perícia relativa ao suporte da aprendizagem dos professores em formação e realizam um bom trabalho em seus devidos espaços, seus tempos de trabalho nos programas são limitados e, a cada novo ano acadêmico, uma nova turma de estudantes de pós-graduação adentra no departamento com um conhecimento muito restrito das especificidades do trabalho que foi desenvolvido até então, de tal modo que a introdução desses indivíduos num processo em andamento de renovação do programa recomeça mais uma vez. Em função de esses supervisores virem de todo mundo a Madison para terminar os seus estudos, eles, com frequência, não estão familiarizados com as escolas locais; e o modo como seus papéis geralmente são estruturados leva-os a trabalhar em várias, isto é, uma porção de escolas diferentes num dado a qualquer momento, e mesmo em escolas um tanto quanto diferentes entre si a cada semestre. Além disso, à exceção de dois programas de formação para educação básica em escolas de desenvolvimento profissional, nos quais os estudantes permanecem com o mesmo supervisor acadêmico e as mesmas duas escolas durante quatro semestres, um supervisor é responsável em cada semestre por trabalhar com 
um diferente grupo de estagiários ou estudantes ainda em formação, situação que torna mais difícil o aprofundamento do processo de supervisão (ZEICHNER \& MILLER, 1997).

Mesmo nos programas da UW-Madison e de outras instituições, em que o corpo docente e/ou técnico participa de uma maneira significativa, lecionando disciplinas da universidade nos cursos de formação de professores e na supervisão de estudantes em seus campos de estágio, a desconexão entre a formação de professores no âmbito acadêmico e nas escolas tem sido um problema perene (Vick, 2006). Já tem sido claramente documentada há muitos anos (por exemplo, Clifford \& Guthrie, 1988; Goodlad, 1990; Labaree, 2004), que existem poucos incentivos para professores efetivos investirem algum tempo na coordenação entre os componentes acadêmicos e os que ocorrem a campo na formação de professores e, sobretudo, para tutorar e monitorar o trabalho dos supervisores a campo. As instituições têm, com efeito, utilizado às vezes um corpo de professores colaboradores (isto é, professores recentemente aposentados) para fazer o trabalho de supervisão de estudantes em suas respectivas escolas de atuação, mas, muito constantemente, esses dedicados e competentes indivíduos não têm poder decisório sobre os programas de formação de professores, assim como não estão em contato direto com a parte do programa desenvolvida na universidade (BULLOUGH et al, 1997; BULLOUGH et al, 2004; COMBLETH \& ELLSWORTH, 1994; ZEICHNER, 2002).

Em geral, o processo de colocação de estagiários pelas faculdades e pelas universidades é "terceirizado"; ou seja, fica sob o encargo de um escritório administrativo central ao invés dos departamentos; com isso, o que determinará os lugares para os quais irão os professores em formação para suas experiências escolares é a disponibilidade de professores regentes na escola [para receber estagiários] ou considerações de ordem administrativa, ao invés, do que é melhor para a aprendizagem de professores principiantes. (ZEICHNER, 1996).

Do lado da escola, os professores convidados a tutorar os estagiários colocados em suas salas de aula por períodos variados de tempo durante as práticas educativas, estágio e (eventual) período de estágio de imersão, têm que fazer o trabalho da formação do professor, além de atenderem plenamente suas responsabilidades dentro da sala de aula; se recompensados por este trabalho, a remuneração é proporcionalmente inferior ao salário mínimo.

Na visão tradicional sobre experiência de campo, já dominante por muitos anos, espera-se que esses professores formadores das escolas ofereçam um espaço para os estagiários praticarem o ensino e, geralmente, não se Ihes dá a preparação e o suporte que eles precisariam ter (VALENCIA et al, 2009) para implementar uma concepção de tutoria mais ativa e educativa (CARROL, 2007; MARGOLIS, 2007). Como assinalam Gorodetsky, Barak e Harari (2007), mesmo com a atual onda de parcerias escola-universidade na formação de professores, as faculdades e as universidades continuam a manter a hegemonia 
Repensando as conexões entre a formação na universidade e as experiências de campo na formação de professores em faculdades e universidades

sobre a construção e a disseminação do conhecimento, ao passo que as escolas permanecem na posição de "campos de prática" (BARAB \& DUFFY, 2000), nos quais estagiários devem tentar realizar as práticas ensinadas na universidade.

\section{A tradicional divisão entre a formação de professores na universidade e nas escolas}

No modelo, historicamente, dominante da "aplicação da teoria" na formação inicial de professores nos Estados Unidos, supõe-se que os professores em formação devam aprender as teorias na universidade para, depois, ir às escolas e praticar ou aplicar o que foi aprendido no espaço acadêmico (KORTHAGEN \& KESSEKS, 1999; TOM, 1997). De maneira alternativa, em alguns modelos de antecipação de entrada de professores em formação na escola, em que há muito pouco trabalho de curso antes que os candidatos assumam total responsabilidade por uma sala de aula, supõe-se que o que a maior parte do professores principiantes precisa aprender sobre o ensino pode ser aprendida no exercício da função, na prática mesmo, isso porque o papel da universidade pode, nesse processo, ser minimizado, sem que demande grandes perdas (GROSSMAN \& LOEB, 2008).

Embora haja um crescente consenso de que muito do que os professores precisam aprender deve ser aprendido na e a partir da prática, ao invés de na preparação para a prática (BALL \& COHEN; HAMERNESS et al, 2005), existe ainda grande desacordo com relação às condições necessárias para que a aprendizagem docente aprendida dentro e a partir da prática seja de fato educativa e duradoura. Por exemplo, o ponto em que um professor deve tornarse o professor regente é uma questão sobre a qual tem havido também muita discordância (STODDART \& FLODEN, 1996). Aqueles que advogam pelos programas de "antecipação de entrada" têm argumentado que, com uma cuidadosa seleção e um mínimo de experiência em estágio profissional, indivíduos podem tornar-se professores regentes de modo razoavelmente rápido, aprendendo o que precisam aprender sobre o ensino desde que bem assessorados (GROSSMAN \& LOEB, 2008). Já outros defendem uma entrada mais gradual no ensino, supondo que a responsabilidade por uma classe de alunos vem depois ou em conjunto com uma substantiva integralização curricular uma extensa passagem pelo estágio ou residência docente, sob a orientação cuidadosa de um professor que é responsável pela turma. Os modelos de residência docente - foco de um investimento federal de 100 milhões de dólares, no primeiro ano de administração de Barack Obama - são exemplos de programas que representam essa posição de entrada gradual (BERRY, MONTGOMERY \& SNYDER, 2008).

Um problema perene em programas tradicionais de formação de professores mantidos por faculdades e universidades tem sido a da falta de conexão entre os cursos de formação de professores nessas unidades e o campo da 
prática. Embora a maior parte dos programas universitários de formação de professores inclua, agora, múltiplos campos de experiência em toda a sua extensão e situem, geralmente, as experiências de campo em algum tipo de parceria escola-universidade (por exemplo, escolas de desenvolvimento profissional escolas parceiras), a desconexão entre o que é ensinado aos estudantes nos cursos acadêmicos e suas oportunidades de aprendizagem para levar a termo tais práticas em suas respectivas escolas não raramente é muito grande, inclusive nas escolas parceiras e nas de desenvolvimento profissional (BULLOUGH et al, 1997; BULLOUGH et al; 1999; ZEICHNER, 2007).

É muito comum, por exemplo, que os professores regentes, com os quais os estudantes trabalham durante sua passagem pela escola, saibam muito pouco sobre a especificidade das disciplinas de metodologia e fundamentos que seus estagiários completaram na universidade, assim como aqueles que lecionam na universidade pouco sabem sobre as práticas específicas utilizadas em classes da Educação Básica, nas quais seus estudantes estão alocados. Os estagiários raramente têm oportunidade de observar, experimentar e receber uma resposta pontual sobre como ensinam e aplicam os métodos aprendidos na universidade. Ainda que as práticas advogadas nas disciplinas na universidade existam nas salas de aula em que os estagiários lecionam, eles não têm necessariamente acesso ao pensamento e ao processo decisório de seus tutores (HAMMERNESS et al, 2005; ZEICHNER, 1996) que, usualmente, são mal remunerados pelo complexo e difícil trabalho que se espera que façam para orientar os estagiários. Darling-Hammond (2009), em seus estudos, por exemplo, não deixa de referir-se à falta de conexão entre a formação na universidade e o campo da prática como sendo o calcanhar de Aquiles da formação de professores.

Embora muitos programas incluam experiências de campo ao longo de seus currículos, o tempo que os futuros professores passam nas escolas com frequência não é devidamente planejado da mesma forma que se faz com as disciplinas na universidade que possuem "currículo técnico" (TURNEY et al, 1985). À exceção de algumas tarefas das disciplinas de metodologia que os estudantes devem que completar nas escolas, cabe aos estagiários e a seus respectivos professores regentes a elaboração do seu plano de trabalho sem muita orientação e/ou conexão com as disciplinas da universidade, do que se depreende, rotineiramente, que boas práticas de ensino são antes percebidas do que ensinadas (DARLING-HAMMOND, 2009; VALENCIA et al, 2009).

Pesquisas têm mostrado claramente que as experiências de campo constituem importantes ocasiões para que se efetive a aprendizagem docente mais do que meramente épocas nas quais os futuros professores podem demonstrar ou aplicar coisas previamente aprendidas (ZEICHNER, 1996). Rosean e Florio-Ruane (2008), por exemplo, discutem como as suposições tomadas como corretas, acerca dos propósitos da experiência de campo na formação de professores, limitam o valor delas como experiência de aprendizagem docente; 
Repensando as conexões entre a formação na universidade e as experiências de campo na formação de professores em faculdades e universidades

ao mesmo tempo, os autores oferecem ideias para se repensar a experiência de campo como sendo ambientes mais produtivos de aprendizagem.

As propostas de Cochran-Smith e Lytle (2009) sobre a utilização da prática de ensino como um lugar para a problematização constitui outro exemplo de mudança de paradigma no pensamento sobre o papel da experiência de campo na formação de professores. Dois dos mais aprofundados estudos nacionais sobre a formação de professores nos Estados Unidos mostraram que experiências de campo cuidadosamente construídas - que sejam coordenadas com os cursos acadêmicos - são mais influentes e efetivas no apoio à docência do que experiências de campo não orientadas e desconectadas, as quais têm sido historicamente dominantes na formação de professores norte-americanos (DARLING- HAMMOND, 2006; TATTO, 1996). Há uma infinidade de estudos demonstrando, já há alguns anos, os obstáculos à aprendizagem do professor em formação associados com o tradicional modelo de experiência de campo precariamente planejado e monitorado (por exemplo, FEIMAN-NEMSER \& BUHMANN, 1985; GRIFFIN et al, 1983; STONES \& MORRIS, 1977; ZEICHNER, 1996).

Ao longo dos anos, formadores de professores têm tentado uma variedade de abordagens para fortalecer as conexões entre formação de professores no campo e no âmbito acadêmico; alguns estudiosos vêm argumentando que experiências de inserção [clínica] deveriam ser o foco central da formação inicial de professores, a partir da qual tudo mais emanaria (TURNEY et al, 1985; BALL \& FORZANI, 2009). Essas estratégias incluem a criação de escolas de aplicação nas faculdades e nas universidades, em que determinadas abordagens de ensino podem ser demonstradas, praticadas sob a orientação dos docentes e técnicos da universidade (FRASER, 2007). Na impossibilidade da abertura de escolas em espaços acadêmicos, formadores de professores criaram, nas universidades, laboratórios de prática de ensino, nos quais habilidades e práticas de ensino específicas são ensinadas (BERLINER, 1985; GROSSMAN, 2005; METCALF \& KAHLICH, 1996); buscando modelar, nas disciplinas da universidade, as práticas que eles esperam que seus estudantes usem em suas salas de aula da Educação Básica. Esses professores têm criado simulações de situações de sala de aula ou reunido registros de prática de sala de aula, utilizando-se de ferramentas como a hipermídia, casos escritos e multimídia, assim como criado tarefas que os estudantes devem implementar nas escolas em que estão alocados. Vale assinalar ainda que, em alguns programas, os mesmos profissionais servem como professores de metodologia e supervisores de estágio (por exemplo, Cohn, 1981).

Desde o início dos anos de 1970, John Goodlad tem argumentado a favor da criação de Centros Pedagógicos nas faculdades e nas universidades como estruturas em que a formação de professores deveria se situar. De acordo com Goodlad (2004a), essa estrutura 
[...] é um espaço que reúne e mistura harmoniosa e coerentemente os três ingredientes essenciais da formação de professores: a educação geral e livre; o estudo da prática educativa e o exercício orientado da arte, da ciência e da habilidade do ensinar (p. 2-3).

$\mathrm{Na}$ atualidade, existe um número de instituições associadas a National Network for Educational Renewal (http://www.nnerpartnership.org/), a NNER [Rede Nacional pela Renovação Educacional] que implementaram o conceito de Centro Pedagógico, como é o caso da Montclair State University e da Brigham Young University (PATTERSON, MICHELLI \& PACHECO, 1999). Ainda que em alguns aspectos essas estruturas tenham criado um espaço mais neutro no qual os componentes da formação de professores (à exceção da comunidade mais ampla) podem se unir numa relação de benefício e respeito mútuo; ao meu entender, como um observador externo, as universidades e as escolas, nessas parcerias, têm mantido separadas as suas respectivas culturas e formas singulares de discurso, o que tem tornado o aspecto institucional do processo de renovação limitado. Os tipos de colaboração discutidos, neste trabalho, focam mais na criação de novas espécies de papéis para os formadores de professores e nos meios de aproximar conhecimento acadêmico, profissional e derivado da comunidade no processo de formação de professores, do que na mudança estrutural das instituições de formação de professores.

Neste texto, utilizarei o conceito de "terceiro espaço" como uma lente para discutir vários tipos de cruzamentos de fronteira entre universidade e escola atualmente em desenvolvimento em programas de formação de professores nos Estados Unidos. Com a emergência de uma formação de professores focada na escola, nos modelos de residência docente que têm sido promovidos pela atual administração federal dos Estados Unidos (BERRY et al, 2008), as experiências [clínicas] práticas e o ensino da prática na formação inicial de professores estão recebendo uma significativa atenção e, por conseguinte, os meios tradicionais de organizar a formação de professores nas universidades e no campo têm sido repensados. ${ }^{1}$

\section{Criando novos espaços híbridos: relacionando conhecimento profissio- nal e acadêmico}

A ideia de um terceiro espaço vem da teoria do hibridismo e reconhece que indivíduos extraem, de múltiplos discursos, elementos para fazer um sentido de mundo (BHABBA, 1990). Terceiros espaços envolvem uma rejeição das binaridades tais como entre o conhecimento prático profissional e o conhecimento acadêmico, entre a teoria e a prática, assim como envolve a integração, de novas maneiras, do que comumente é visto como discursos concorrentes em que uma perspectiva do isso ou aquilo é transformada num ponto de vista do tanto isso, quanto aquilo. O conceito de terceiro espaço tem sido usado em campos como a geografia, as ciências humanas, os estudos pós-coloniais, os 
Repensando as conexões entre a formação na universidade e as experiências de campo na formação de professores em faculdades e universidades

estudos feministas e, mais recentemente, na educação (por exemplo, GUTIERREZ, 2008; MOJE et al, 2004; SOJA, 1996), incluindo-se aí a formação de professores (COCHRAN-SMITH \& LYTLE, 1999). ${ }^{2}$

O uso que faço, no presente texto, do conceito de terceiro espaço diz respeito à criação de espaços híbridos nos programas de formação inicial de professores que reúnem professores da Educação Básica e do Ensino Superior, e conhecimento prático profissional e acadêmico em novas formas para aprimorar a aprendizagem dos futuros professores. Contrários à desconexão tradicional entre escola e universidade e à valorização do conhecimento acadêmico como a fonte de autoridade do conhecimento para a aprendizagem sobre o ensino, próprio dos modelos tradicionais de formação de professores das escolas normais superiores e das universidades (SMAGORINSKY, COOK e JOHNSON, 2003), os terceiros espaços reúnem o conhecimento prático ao acadêmico de modos menos hierárquicos, tendo em vista a criação de novas oportunidades de aprendizagem para professores em formação. Gutierrez (2008, p.152) afirma que um terceiro espaço é "um espaço transformativo no qual o potencial para uma forma expandida de aprendizagem e desenvolvimento de um novo conhecimento é aumentado". A discussão de Gorodetsky e Barak (2008) de "comunidades [ou zonas] de transição" nas parcerias entre escolas e universidades na formação de professores - que constituem uma espécie de terceiro espaço - traz o argumento de que esses espaços híbridos incentivam um status mais igualitário para seus participantes, diferentemente do que acontece nas parcerias convencionais entre escola e universidade.

Da perspectiva das faculdades e das universidades, a solução para a desconexão entre universidade e escolas na formação de professores e na formação profissional continuada para professores da Educação Básica tem sido, habitualmente, tentar perceber maneiras melhores de trazer o saber acadêmico das faculdades e das universidades para os professores da Educação Básica. Esse tem sido um modelo de fora para dentro, no qual o saber está primordialmente entre os acadêmicos e não entre os professores da Educação Básica (ZEICHNER, 1995). A criação de terceiros espaços na formação de professores envolve uma relação mais equilibrada e dialética entre o conhecimento acadêmico e o da prática profissional, a fim de dar apoio para a aprendizagem dos professores em formação.

\section{Cruzamentos de fronteira e a criação de terceiros espaços na formação de professores}

Descreverei, em continuidade, uma série de diferentes tipos de cruzamentos de fronteira que têm ocorrido nos últimos anos em alguns programas de formação de professores em faculdades e universidades, num esforço de reunião de conhecimento prático profissional ao acadêmico, de um modo mais sinérgico no acompanhamento da aprendizagem dos professores em formação. Embora estas experiências de mudança da epistemologia da formação docente 
inicial a partir de um lugar no qual o conhecimento acadêmico é tomado como a fonte primeira de conhecimento sobre o ensino, para uma situação na qual o conhecimento acadêmico e o conhecimento dos professores experientes da Educação Básica gozam da mesma importância, isso não configura uma lista detalhada de todas as instituições nas quais esse trabalho tem se desenvolvido. Meu objetivo é ilustrar diferentes tipos de espaços híbridos que têm sido criados na formação de professores, citando apenas alguns exemplos de cada uma dessas formas com as quais tenho me familiarizado.

\section{Reunindo professores da Educação Básica e seu conhecimento nas dis- ciplinas acadêmicas e nas experiências de campo}

Por muitos anos, tem sido comum, para faculdades e universidades, contratar educadores da Educação Básica para uma função adjunta de ensinar partes ou segmentos das disciplinas exigidas em programas de formação inicial de professores. Além desses contratos de curta duração, um número de programas tem contratado professores por períodos mais prolongados para que se envolvam em atividades de ensino e de co-regência, na supervisão de estudantes e na participação, na renovação e na avaliação de programas em andamento.

Os cargos de professor associado na Simon Fraser University, na Columbia Britânica e na Brigham Young University (BEYNON et al, 2004; BULLOUGH et al, 2004), assim como o cargo de professor residente na Universidade de Wisconsin-Milwaukee (POST et al, 2006), são exemplos dessa abordagem.

No caso da Universidade de Wisconsin-Milwaukee, o Programa de Professores em Residência procura criar uma ligação mais forte entre a preparação acadêmica do professor e o saber proveniente da experiência dos professores das escolas. Professores com evidência de alto nível de competência em sala de aula passam dois anos trabalhando todos os aspectos da formação inicial de professores, incluindo a seleção de estudantes, a formação geral em educação e específica nas áreas, a sequência da educação profissional, a avaliação de programas em andamento e os esforços de renovação, até o acompanhamento de egressos em seus primeiros anos de profissão docente. Durante sua residência bienal, esses professores participam de seminários, que pretendem desenvolver as habilidades de liderança do professor, sendo que, depois de sua residência, eles voltam às escolas públicas de Milwaukee. Tive a oportunidade de entrevistar vários professores universitários e residentes ao longo dos dois anos que passei, recentemente, como avaliador externo para o Projeto Professores para uma Nova Era da Universidade de Wisconsin-Milwaukee e muitos professores, que entrevistei, falaram positivamente sobre o significativo impacto dos professores residentes em seus cursos. 
Repensando as conexões entre a formação na universidade e as experiências de campo na formação de professores em faculdades e universidades

\section{Incorporando representações das práticas dos professores nos cursos das universidades}

Uma alternativa para trazer os professores diretamente às atividades da formação de professores, nas unidades acadêmicas, é a criação de oportunidades para que representações das práticas docentes sejam trazidas aos respectivos cursos. Um exemplo dessa estratégia tem sido incorporar a escrita e a pesquisa de professores da Educação Básica (por exemplo, GALLAS, 2004; GOLDSTONE, 2003; HANSON, 2008) ao currículo da universidade para que os estudantes possam examinar tanto o conhecimento acadêmico como o gerado pelos profissionais no que se refere aos aspectos específicos de ensino. Além de fornecer aos professores em formação insights para a compreensão das complexidades de determinadas práticas docentes, essa estratégia também oferece aos principiantes exemplos de professores capazes de aprender na e com a prática ao longo do tempo.

Há mais de dez anos, a Carnegie Foundation for the Advancement of Teaching trabalha com professores da Educação Básica, nos Estados Unidos, para criar modelos multimídia, em ambientes virtuais, de suas práticas de ensino (POINTER, MACE, 2009). Na sequência disso, um grupo de formadores de professores por todo o país e, com o suporte da Carnegie Foundation, usou os sites dos professores da Educação Básica nos cursos acadêmicos e, desse modo, criaram, com seus estudantes em formação inicial, seus próprios websites multimídia sobre o uso dos sites dos professores da Educação Básica. ${ }^{3}$ Pam Grossman, por exemplo, uma formadora de professores de Stanford, criou um site no qual ela documentou como incorporou o website de uma experiente professora de língua inglesa de uma Escola de Ensino Médio de Los Angeles (Yvonne Divans Hutchinson) em seu curso de metodologia da língua inglesa, na Universidade de Stanford. Um aspecto desse trabalho centrou-se na tarefa de envolver os estudantes em discussões sobre literatura baseadas em textos.

Além de ler literatura acadêmica sobre esse assunto, os estudantes utilizaram também o website de Hutchinson - o qual inclui: imagens de suas principais discussões, com a participação ativa de seus alunos; entrevistas com ela e pronunciamentos de seus alunos, assim como exemplos do trabalho dos estudantes e dos métodos e materiais que Hutchinson usou para preparar seus alunos para a discussão.

Uma alternativa para os professores universitários formadores de professores, que adotam os modelos das práticas dos professores da Educação Básica, é produzir modelos a partir de suas próprias experiências e práticas no ensino fundamental e médio a fim de utilizá-las na prática do ensino superior. Uma experiência nessa linha de trabalho foi realizado por Flessner (2008), que construiu modelos didáticos de seu ensino de matemática para crianças do ensino fundamental (videoclipes, exemplos de trabalho de estudantes), no ensino de matemática nos cursos de graduação da Universidade de Wisconsin- 
Madison. Outro exemplo disso é a documentação estratégica do ensino de matemática elementar com hipermídia de Lampert \& Ball (1998).

\section{Instrução mediada e experiências de campo}

Já faz algum tempo, é comum que professores dos cursos das universidades realizem uma parte ou a totalidade das disciplinas de metodologia em escolas do ensino fundamental e médio. Lecionar uma disciplina dentro da escola não quer dizer necessariamente que essa venha a ser diferente de qualquer outra desenvolvida na universidade. Alguns formadores de professores, no entanto, aproveitam o espaço da escola e estrategicamente conectam suas disciplinas de metodologia, que lecionam no contexto da escola, com as práticas e o conhecimento especializado dos professores nessas escolas. Para exemplificar isso, podemos tomar o trabalho desenvolvido, nos últimos anos, na University of Washington-Seattle, em que os professores de metodologia dos programas de formação de professores para educação básica oferecem uma parcela de suas disciplinas em instituições de Educação Básica parceiras. Motivados pelos resultados obtidos em suas próprias pesquisas, que mostram que seus estudantes não estão se apropriando das ideias e práticas veiculadas nos espaços acadêmicos, o corpo docente das disciplinas de metodologia comprometeu-se a mediar as lacunas entre os cursos acadêmicos e a experiências desses alunos na escola. Como ocorreu no caso do programa de matemática no ensino médio:

[...] os estagiários não tinham uma visão ou um modelo concreto de como é uma sala de aula onde as práticas promovidas foram usadas para ensinar matemática para compreensão (CAMPBELL, 2008, p. 09).

De cada dois encontros por semana da disciplina de metodologia do ensino de matemática, na formação de professores para o ensino secundário, um acontecia numa escola de ensino médio local, em que professores empregavam práticas similares às ensinadas na disciplina acadêmica. A turma e o professor da universidade observaram a mesma turma de nono ano a cada semana com reuniões com o professor logo após cada aula observada.

O trabalho em Seattle é semelhante às atividades em execução na Michigan State University, durante os anos de 1990, quando esforços foram feitos para redefinir o papel dos professores colaboradores em escolas de desenvolvimento profissional selecionadas, de modo a que desempenhassem um papel mais ativo na demonstração e na orientação dos estagiários e dos préestagiários na análise de práticas de ensino específicas. Num exemplo desse tipo de trabalho que foi documentado pelos pesquisadores da Michigan State, um grupo de estudantes de metodologia do ensino de matemática no ensino fundamental passou uma semana na sala de aula de Kathy Beasley, para observála lecionando matemática. Antes e depois de cada aula de matemática, o grupo 
Repensando as conexões entre a formação na universidade e as experiências de campo na formação de professores em faculdades e universidades

de estudantes de metodologia do ensino de matemática encontrava com Kathy e sua estagiária da Michigan State University para analisar as práticas que foram observadas (Feiman-Nemser \& Beasley, 2007).

Um terceiro exemplo de supervisão mediada e experiências de campo é o trabalho de mais de uma década na University of Wisconsin-Madison, na qual uma disciplina de Métodos de Alfabetização tem se dado em uma escola de desenvolvimento profissional (EDP) associada à Universidade. Nesse caso, o coordenador interno da EDP trabalha com o professor de metodologia que é geralmente um estudante de doutorado, para ir além do programa da disciplina e conectar os conceitos e as práticas ensinadas na disciplina com o conhecimento especializado que existe na escola. Uma vez que os alunos de metodologia estão estudando sobre determinadas abordagens da alfabetização, tal como a instrução equilibrada, eles têm a chance de observar e interagir com professores que são especialistas nessas práticas. Às vezes, a turma segue em "grupos de análise técnica" (TROEN et al, 1997) para uma sala de aula com o propósito de ver um professor envolvido numa determinada prática; em continuidade, reúnem-se com aquele professor após a observação; às vezes um professor da escola vai à disciplina de metodologia discutir o seu trabalho, frequentemente levando artefatos de sua prática, tais como trabalhos de alunos. Em ambos os casos, há um esforço deliberado para conectar estrategicamente conhecimento acadêmico e conhecimento da prática profissional em vista de um melhor acompanhamento da aprendizagem dos professores em formação acerca de como levar a termo práticas de ensino específicas defendidas nos cursos de metodologia.

\section{Formadores híbridos de professores}

Algumas instituições de formação de professores estabeleceram, em seu corpo docente, posições técnicas, em que o trabalho dos formadores de professores acontece tanto nas escolas de ensino fundamental e de ensino médio, quanto nas faculdades e nas universidades. Comecei minha carreira de formador universitário de professores numa posição limítrofe em meados dos anos de 1970 (Howey \& Zimpher, 2006), como líder de equipe no projeto National Teacher Corps, em Syracuse, estado de New York. Minha função, como líder de equipe, era supervisionar o trabalho de uma turma de estagiários vinculados a um programa de formação docente de curta duração (dois anos) numa escola pública de ensino fundamental. Como líder de equipe, eu precisava estar intimamente familiarizado com o que era estudado no curso e com o trabalho comunitário dos estagiários, já que supervisionava suas experiências de campo num período de dois anos e tinha que funcionar como membro do corpo docente tanto da escola na qual eu lecionava, como também da universidade.

Há diferentes tipos de formadores híbridos de professores existentes por toda a nação. Tal variedade inclui cargos docentes de supervisão (em geral, não efetivos) que trabalham para construir parcerias com escolas locais, cujo 
foco é a formação inicial de professores e, às vezes, o desenvolvimento profissional continuado de professores (BOYLE-BAUSE \& MCINTYRE, 2008). Também abarcam cargos nos quais docentes atuam nos campos de estágio no ensino fundamental ou no médio, onde fazem a colocação dos estagiários na escola, como também supervisionam sua experiência na escola.

Durante três anos na década de 1990, eu servi como o elo da faculdade com duas escolas elementares de desenvolvimento profissional das quatro afiliadas ao programa experimental "Ensino para a diversidade", da UW-Madison (Ladson-Billings, 1999). Durante esse período, eu recebi créditos para uma disciplina ao ano para lecionar, junto com um supervisor da universidade sediado em cada escola, um seminário semanal para estagiários do programa "Ensino para a diversidade", no qual ajudávamos os estagiários a analisar seus trabalhos na escola em relação a uma variedade de conceitos e perspectivas que lhes eram introduzidas nas disciplinas acadêmicas. Trabalhei também com a equipe da escola para organizar e, às vezes, participar nas atividades de ensino de desenvolvimento profissional que foram planejadas com e para a equipe da escola, com os professores em formação e, também, às vezes, com os pais. Ao trabalhar como docente da faculdade no Ensino Fundamental e no Departamento de Currículo e Instrução, eu tinha uma sala nas escolas e, periodicamente, participava das reuniões que, nelas, se realizavam. Essas posições limítrofes aumentaram muito pelo país afora junto com o crescimento do movimento de escolas de desenvolvimento profissional desde meados da década de 1980 (ZEICHNER, 2007).

\section{Incorporando conhecimento das comunidades à formação inicial de pro- fessores}

Por muitos anos, formadores de professores têm defendido a ampliação do locus da formação inicial de professores da universidade e escolas para as comunidades mais abrangentes nas quais as escolas estão situadas (por exemplo, CUBAN, 1969; FLOWERS et al, 1948). Embora algumas experiências de campo comunitárias em formação docente tenham se centrado na aprendizagem do trabalho e na tutoria dos alunos, e não na aproximação desses com os adultos numa comunidade mais abrangente, outras linhas de trabalho na formação de professores em comunidades têm focado estrategicamente na utilização do saber que existe, nestes locais, para educar os aspirantes a professores sobre como serem bem sucedidos nas suas respectivas comunidades (por exemplo, BOYLE-BAISE \& McINTIRE, 2008; MAHAN, 1982; SLEETER, 2008a; ZEICHNER \& MELNICK, 1996). O trabalho de uma década de Barbara Seidel e Gloria Friend (2002), em Columbus, Ohio, é um modelo dessa abordagem à aprendizagem baseada na comunidade. Nesse trabalho, os aspirantes a professor para o ensino fundamental, da Ohio State University, formaram duplas com adultos de igual status num programa educacional da Igreja Batista Afroamericana, sendo assim, os pesquisadores puderam documentar o impac- 
Repensando as conexões entre a formação na universidade e as experiências de campo na formação de professores em faculdades e universidades

to dessa equalização das relações com os adultos no desenvolvimento das competências culturais dos professores em formação.

Por fim, o trabalho dos formadores de professores na University of Massachusetts-Boston ilustra ainda outra abordagem de utilização do saber das comunidades na formação de professores. Nesse caso, os membros das comunidades foram usados como recursos para educar o corpo docente a respeito das comunidades para as quais ela estava preparando professores para ensinar (KOERNER \& ABDUL-TAWWAB, 2006).

\section{Conclusão}

Desde os primórdios dos programas de formação de professores em faculdades e universidades nos Estados Unidos, pesquisadores têm argumentado contra experiências de campo não orientadas em prol de experiências planejadas e propositivas na escola, baseadas na qualidade da aprendizagem docente que a elas está associada (por exemplo, Dewey, 1904). Neste artigo, busquei apresentar e discutir uma série de esforços atuais, nos Estados Unidos, para diminuir as lacunas entre a formação docente que acontece na universidade e aquela que se dá na escola, assim como as lacunas entre essas duas e as comunidades mais amplas nas quais as escolas, as faculdades e as universidades existem.

Esses esforços envolvem uma mudança na epistemologia da formação do professor, que passa de uma situação em que o conhecimento acadêmico é visto como a fonte legítima do conhecimento sobre o ensino para outra em que diferentes aspectos do saber que existe nas escolas e nas comunidades são incorporados à formação de professores e coexistem num plano mais igualitário com o conhecimento acadêmico. Argumentou-se que essa visão mais ampla sobre os diferentes saberes, que são necessários para formar professores, expande as oportunidades de aprendizagem docente na medida em que novas sinergias são criadas por meio do jogo interativo entre conhecimentos das mais diferentes fontes. Uma pesquisa recente, usando a teoria da atividade sobre transições escola-trabalho e interações de diferentes sistemas de atividade, embasa essa afirmação (por exemplo, TUOMI-GROHN, 2007). Ainda que a criação desse tipo de espaços híbridos na formação de professores não aborde diretamente os problemas institucionais e culturais que persistentemente minam a qualidade da formação de professores em faculdades, universidades e escolas há muitos anos (por exemplo, seu baixo status, a falta de recompensa por um bom trabalho na formação do professor, a falta de financiamento adequado), ela cria espaços para a aprendizagem dos professores em formação de modo que tirem vantagem das múltiplas fontes de saber que podem, eventualmente, embasar um ensino de qualidade. 
Embora a pesquisa de alta qualidade a respeito do impacto de várias formas de ensino na universidade e acerca das experiências de campo na escola e na comunidade sobre as perspectivas e as práticas dos futuros professores seja razoavelmente limitada, (por exemplo, CLIFT \& BRADY; FLODEN, 2005), alguns estudos começaram a documentar o impacto de determinados tipos de experiências e programas de formação docente sobre o modo como influenciam a aprendizagem dos futuros professores no rumo que se deseja. Darling-Hammond et al (2006) e Zeichner \& Conklin (2008) concluíram que a pesquisa existente sobre programas exemplares de formação de professores mostra que, naqueles em que a experiência de campo é cuidadosamente coordenada com o que se estuda na universidade e é cuidadosamente supervisionada, os formadores de professores estão mais capacitados a alcançar suas metas na preparação de professores para atuarem de forma bem sucedida em práticas de ensino complexas. Em outro exemplo, Campbell (2008) relata que, na University of Washington-Seattle, onde os estagiários participaram sob supervisão mediada em seu programa de certificação em matemática, eles desenvolveram um entendimento muito mais profundo das práticas de ensino promovidas e foram mais bem sucedidos no desenvolvimento dessas práticas em diversas escolas públicas de ensino médio.

O crescente foco atual no repensar e na reformulação da conexão entre as disciplinas da formação inicial de professores nas faculdades e nas universidades e as escolas da rede e as comunidades para as quais esses alunos estão sendo preparados é um sinal otimista de que o tradicional modelo distanciado e desconectado da formação inicial baseada na universidade está em pleno esfacelamento. É necessário ainda que as faculdades e as universidades, assim como as escolas da Educação Básica comecem a pensar como melhor reconhecer e recompensar os membros de seu corpo docente e técnico que realizam de maneira exemplar nos espaços híbridos criados ou, então, o impacto de seu trabalho será mínimo no campo como um todo.

A contínua falta de recompensa e reconhecimento pelo trabalho bem feito na formação de professores pelos docentes efetivos nas universidades voltadas à pesquisa resultou numa maior dependência dos programas de formação docente sobre a atuação dos profissionais de supervisão e dos alunos de pósgraduação e no abandono desse trabalho por muitos professores pesquisadores efetivos (BULLOUGH et al., 1997; GOODLAD, 1994). Embora esses estudantes de pós-graduação e os supervisores tenham trazido muitas coisas positivas para os programas universitários de formação de professores a partir de suas recentes experiências nas escolas, o tipo de transformação na epistemologia da formação do professor discutida, neste artigo, não pode ser realizada em universidades voltadas para a pesquisa sem o direto envolvimento dos professores efetivos. A fim de que esses indivíduos se envolvam, os professores efetivos mais antigos e a administração precisam assumir a liderança na criação das condições, nas quais os professores sejam recompensados pelo seu engajamento e pela criação e pela manutenção de programas exemplares de formação de professores. 
Além disso, é importante que, no atual clima de redução consistente dos orçamentos de faculdades e universidades (LYALL \& SELL, 2006), a formação de professores receba uma parcela justa dos recursos institucionais, a fim de fornecer programas de alta qualidade com rigorosos e, cuidadosamente, planejados componentes de inserção na prática. O tipo de trabalho descrito neste artigo não pode ser bem realizado em larga escala, com carência de recursos pela qual a formação de professores - frequentemente - passa nas faculdades e nas universidades.

Muitos exemplos de cruzamento de fronteira, que mencionei neste estudo, estão situados em universidades focadas na pesquisa e/ou foram apoiadas por algum financiamento externo É importante pensar ainda como criar tais práticas híbridas em todos os tipos de programas de formação de professores, inclusive os de "antecipação de entrada", em diferentes tipos de instituições formadoras de professores e com financiamento continuado.

Atualmente, existe um montante de recursos que estão sendo destinados a atender mecanismos elaborados de avaliação (accountability) para monitorar o atendimento e a conformidade das instituições de formação de professores aos padrões exigidos pelo Estado. É evidente que muito dessa atividade de monitoramento não aborda ou sequer contribui para o aprimoramento da qualidade dos programas de formação de professores (JOHNSON et al, 2005; SLEETER, 2008b; ZEICHNER, 2008) e que um impacto mais significativo, para melhorar a qualidade dos programas e a aprendizagem da docência, pode ser obtido através do desenvolvimento de sistemas mais dinâmicos e relevantes de avaliação e pela realocação de boa parte do dinheiro, na atualidade, gasto no monitoramento burocrático e hiper-racionalizado dos programas ${ }^{4}$ para apoiar os tipos de conexões escola-universidade e comunidade apresentados neste artigo. Existe evidência empírica de que os recursos humanos e financeiros que as instituições de formação de professores tiveram que alocar para produzir relatórios aos estados e às agências de acreditação sobre os seus programas, desviaram a atenção dos formadores de professores no que se refere à criação das práticas inovadoras discutidas neste artigo (KORNFELD et al, 2007; RENNETTARIEV, 2008). A alocação de recursos financeiros competitivos para desenvolver parcerias escola-universidade-comunidade para a formação de professores com alta qualidade, tais como os exemplos, aqui, destacados, seria certamente um uso melhor de recursos do que está ocorrendo neste momento. ${ }^{5}$

Outra maneira de apoiar o desenvolvimento e o aprimoramento contínuo dessas práticas, numa variedade de instituições e programas, é dar suporte à rede de instituições focadas na criação desses tipos de práticas limítrofes e híbridas. A National Network for Educational Renewal, criada por John Goodlad e seus colegas, em 1986, é um exemplo deste tipo de rede. Ademais, redes como a NNER podem dar oportunidade para que formadores de professores aprendam uns com os outros sobre como criar exemplos bem sucedidos de práticas híbridas numa variedade de contextos, podem fornecer ainda assesso- 
ria técnica e, ao fazer isto, podem ajudar a propiciar oportunidades para o financiamento de algumas dessas iniciativas. Neste momento, a NNER está lançando um projeto centrado na preparação de novos formadores de professores vinculados a esses tipos de práticas híbridas discutidas neste estudo.

Há uma grande dose de impaciência com faculdades e universidades por todo o país com relação àquilo que é percebido como nossa relutância à mudança e ao trabalho com escolas e comunidades de modo mais próximo e respeitoso entre as carreiras docentes (por exemplo, HARTOCOLLIS, 2005). A despeito da complexidade em trazer essa nova epistemologia da formação de professores para o discurso dominante, a menos que estejamos disponíveis para fazê-lo o mais breve possível, a formação de professores em faculdades e universidades talvez deixe de ser a fonte principal de professores para as escolas públicas do país. A explosão de cursos de curta duração e outras instituições, nas quais o papel do corpo docente e técnico das faculdades e universidades é mínimo (HOLLAND, 2004), tornar-se-ão a regra. E isso acontecerá em detrimento tanto da aprendizagem do professor quanto da aprendizagem dos alunos, visto que as oportunidades ampliadas de aprendizagem que são criadas por intermédio do jogo interativo de diferentes fontes de conhecimento não serão realizadas.

\section{Referências}

BALL, D. \& COHEN, D. Developing practice, developing practitioners: Toward a practice-based theory of professional education. In: L. Darling-Hammond \& G. Sykes (Eds). Teaching as the learning profession. San Francisco: JosseyBass, p. 3-32, 1999.

BALL, D. \& FORZANI, F. The work of teaching and the challenge for teacher education. Journal of Teacher Education,60 (5), p. 497-511, 2009.

BARAB, S. A. \& DUFFY T. From practice fields to communities of practice. In: D. JONASSEN \& S. LAND (Eds). Theoretical foundations of learning environments. New York: Routledge. p. 25-56, 2000.

BERLINER, D. Laboratory settings and the study of teacher education. Journal of Teacher Education, 36(2), 1985.

BERRY, B.; MONTGOMERY, D. \& SNYDER, J. Urban teacher residency models and institutes of higher education: Implications for teacher preparation. Washington, DC: NCATE. August, 2008.

BERRY, B.; MONTGOMERY, D.; CURTIS, R. HERNANDEZ, M.; WURTZEL, J. \& SNYDER, J. Creating and sustaining urban teacher residencies. Hillsborough, NC: Center for Teaching Quality and the Aspen Institute. August, 2008.

BEYNON, J.; GROUT, J. \& WIDEEN, M. From teacher to teacher educator. Vancouver, CA: Pacific Education Press, 2004. 
Repensando as conexões entre a formação na universidade e as experiências de campo na formação de professores em faculdades e universidades

BHABBA, $\mathrm{H}$. The third space. In: J. RUTHERFORD (Ed.). Identity, community, culture and difference. London: Lawrence and Wishart, p. 207-221, 1990.

BOYLE, BAISE, M. \& MCINTYRE, D. J. What kind of experience? Preparing teachers in PDS or community settings. In M. COCHRAN-SMITH, S. FEIMANNEMSER \& D.J. MCINTYRE (Eds). Handbook of research on teacher education ( $3^{\text {rd }}$ ed.). New York: Routledge, p. 307-330, 2008.

BULLOUGH, R,; HOBBS, S.; KAUCHAK, D.; CROW, N. \& STOKES, D. Longterm PDS development in research universities and the clinicalization of teacher education. Journal of Teacher Education, 48(2), p. 85-93, 1997.

BULLOUGH, R.; BIRRELL, J.; YOUNG, J.; CLARK, D.; ERICKSON, L.; EARLE, R. et al. Paradise unrealized: Teacher education and the costs and benefits of school-university partnerships. Journal of Teacher Education, 50(5), p. 381390, 1999.

BULLOUGH, R.V.; DRAPER, M.J.; SMITH, L.; \& BURRELL, J. Moving beyond collusion: Clinical faculty and university/public school partnership. Teaching and Teacher Education, 20, p. 505-521, 2004.

CAMPBELL, S. S. Mediated field experiences in learning progressive teaching: a design experiment in teacher education. Paper presented at the annual meeting of the American Educational Research Association, New York City, March, 2008.

CARROLL, D. Helping teachers become teacher educators. In: D. CARROLL; H. FEATHERSTONE; J. FEATHERSTONE; S. FEIMAN-NEMSER; \& D. ROOSEVELT (Eds.). Transforming teacher education: reflections from the field. Cambridge, MA: Harvard Education Press. p. 181-202, 2007.

CLIFFORD, G. J. \& GUTHRIE, J. W. Ed school: a brief for professional education. Chicago: University of Chicago Press. 1988.

CLIFT, R. \& BRADY, P. Research on methods courses and field experiences. In M. COCHRAN-SMITH \& K. ZEICHNER (Eds). Studying teacher education. New York: Routledge. p. 309-424, 2005.

COCHRAN-SMITH, M. \& LYTLE, S. Relationships of knowledge and practice: Teacher learning in communities. Review of Research in Education, 24, p. 249-306, 1999.

COCHRAN-SMITH, M. \& LYTLE, S. Inquiry as a stance: practitioner research in the next generation. New York: Teachers College Press. 2009.

COCHRAN-SMITH, M. \& ZEICHNER, K. (Eds). Studying teacher education. New York: Routledge. 2005.

COHN, M. A new supervision model for linking theory to practice. Journal of Teacher Education, 32(3), p. 26-30, 1981. 
Ken Zeichner

CORNBLETH, C. \& ELLSWORTH, J. Teachers in teacher education: clinical faculty roles and relationships. American Educational Research Journal, 31(1), p. 49-70, 1994.

CUBAN, L. The teacher and community. Harvard Educational Review, 39(2), p. 253-272, 1969.

DARLING-HAMMOND, L. Powerful teacher education. San Francisco: JosseyBass. 2006.

DARLING-HAMMOND, L. Teacher education and the American future. February, 2009.

CHARLES W. HUNT LECTURE. Presented at the annual meeting of the American Association of Colleges for Teacher Education, Chicago.

DARLING-HAMMOND, L.; HAMMERNESS, K.; GROSSMAN, P.; RUST, F. \& SHULMAN, L. The design of teacher education programs. In L. Darling-Hammond \& J. Bransford (Eds). Preparing teachers for a changing world. San Francisco: Jossey Bass. p. 390-441, 2005.

DEWEY, J. The relation of theory to practice in education. In: M. Borrowman (Ed.). Teacher education in America: A documentary history. 1904/1965. In: M. Borrowman (Ed.). Teacher education in America: A documentary history. p. 140171. New York: Teachers College Press. (Originally published in the Third yearbook of the National Society for the Study of Education, p. 9-30. 1904/ 1965.

FEATHERSTONE, J. Values and the big university education school. In D. CARROLL, H. FEATHERSTONE, J. FEATHERSTONE, S. FEIMAN-NEMSER, \& D. ROOSEVELT (Eds). Transforming teacher education: reflections from the field. p. 203-220, 2007.

FEIMAN-NEMSER, S. \& BEASLEY, K. Discovering and sharing knowledge: Inventing a new role for cooperating teachers. In D. CARROLL, H. FEATHERSTONE, J. FEATHERSTONE, S. FEIMAN-NEMSER, \& D. ROOSEVELT (Eds). Transforming teacher education: Reflections from the field. p. 139-160. Cambridge, MA: Harvard Education Press. 2007

FEIMAN- NEMSER, S. \& BUCHMANN, M. (1985). Pitfalls of experience in teacher education. Teachers College Record, 87, 49-65.

FLESSNER, R. Living in multiple worlds: utilizing third space theory to reenvision pedagogy in the field of teacher education. Unpublished doctoral dissertation, University of Wisconsin-Madison, 2008.

FLODEN, R.Research on the effects of coursework in the arts and sciences and in the foundations of education. In M. Cochran-Smith \& K. Zeichner (Eds). Studying teacher education, p. 261-308. New York: Routledge, 2005. 
Repensando as conexões entre a formação na universidade e as experiências de campo na formação de professores em faculdades e universidades

FLOWERS, J.G.; PATTERSON, A.; STRATEMEYER, F.; \& LINDSEY, M. School and community laboratory experiences in teacher education. Oneata, N.Y.: American Association of Teachers Colleges, 1948.

FRASER, J. W. Preparing America's teachers: a history. New York: Teachers College Press, 2007

GALLAS, K. (2004) Look, Karen, I'm running like jello: Imagination as a question, a topic, a tool for literacy research and learning. In C. Ballanger (Ed.) Regarding children's words: Teacher research on language and literacy. New York: Teachers College Press. Brookline MA Teacher Researcher Seminar, p. 119-148

GOLDSTONE, L. The mother tongue: The role of parent-teacher communication in helping students reach new standards. In F.E. Meyers and F. Rust (Eds.) Taking action with teacher research. (p. 63-78). Portsmouth, N.H. : Heinemann. Teachers Network Policy Institute, NYC, 2003

GOODLAD, J. Teachers for our nation's schools. San Francisco: Jossey Bass, 1990.

GOODLAD, J. Educational renewal: Better teachers, better schools. San Francisco: Jossey-Bass, 1994.

GOODLAD, J. The National Network for Educational renewal. Phi Delta Kappan, 75(8), 32-38. 2004.

GORODETSKY, M. \& BARAK, J. 2008. The educational-cultural edge: a participative learning environment for co-emergence of personal and institutional growth. Teaching and teacher education, 24,1907-1918, 2008.

GORODETSKY, M.; BARAK, J. \& HARRARI, H. A cultural-ecological edge: a model for a collaborative community of practice. In M. Zellermayer \& E. Munthe (Eds). Teachers learning in communities: International perspectives. (p. 99112). Rotterdam: Sense Publishers, 2007.

GRIFFIN, G. Clinical pre-service teacher education: final report. Austin, TX: Research and Development Center for Teacher Education, University of TexasAustin, 1983.

GROSSMAN, P. Pedagogical approaches in teacher education. In M. CochranSmith \& K. Zeichner. (Eds). Studying teacher education. p. 425-476, 2005.

GROSSMAN, P. \& LOEB, S. (Eds.). Taking stock: an examination of alternative certification. Cambridge, MA: Harvard Education Press, 2008.

GROSSMAN, P.; COMPTON, C.; IGRA, D; RONFELDT, M.;SHAHAN, E. \& WILLIAMSON, P. (2009). Teaching practice: a cross-professional perspective. Teachers College Record, 111(9). Accessed from tcrecord.org, July 1, 2009.

GUTIERREZ, K. Developing sociocultural literacy in the third space. Reading Research Quarterly, 43(2), 148-164, 2008. 
Ken Zeichner

HAMNERNESS, K.; DARLING-HAMMOND, L. \& BRANSFORD, J. How teachers learn and develop. In L. Darling-Hammond \& J. Bransford (Eds). Preparing teachers for a changing world. p. 358-389. San Francisco: Jossey Bass, 2005.

HANSON, D.Crossing the bridges of culture, color and language. In C. CAROBRUCE, M. KLEHR, \& K. ZEICHNER (Eds.) Using action research to create equitable classrooms. Thousand Oaks: Corwin, 2008.

HARTOCOLTIS, A. Who needs education schools? New York Times (Education Life), p.. 24-28, 2003.

HOLLAND, R.G. To build a better teacher: the emergence of a competitive education industry. Westport, CT: Prager, 2004.

HOWEY, K. \& ZIMPHER, N. (Eds.). Boundary spanners. Washington, DC: American Association of State Colleges and Universities, 2006.

JOHNSON, D.; JOHNSON, B.; FARENGA, S. \& NESS, D. Trivializing teacher education: the accreditation squeeze. Lanham, MD: Roman \& Littlefield, 2005.

KOERNER, M.E. \& ABDUL-TAWWAB, N. Using community as a resource for teacher education: A case study. Equity and Excellence in Education, 39(1), 37-46, 2006.

KORNFELD, J.; GRADY, K. MARKER, P. \& RUDDELL, M. Caught in the current: A self-study of state-mandated compliance in a teacher education program. Teachers College Record, 109(8), 1902-1930, 2007.

KORTHAGEN, F. \& KESSELS, J. Linking theory and practice: Changing the pedagogy of teacher education. Educational Researcher, 28(3), 4-17, 1999.

LADSON-BILLINGS, G. Crossing over to Canaan: the journey of new teachers in diverse classrooms. San Francisco: Jossey Bass, 2001.

LABAREE, D. The trouble with ed schools. New Haven: Yale University Press 2004.

LAMPERT, M.\& BALL,M. Mathematics teaching and multimedia: investigations of real practice. New York: Teachers College Press, 1998.

LYALL, K. \& SELL, K. The true genius of America at risk: Are we losing our public universities to de facto privatization? Westport CT: Prager, 2006.

MAHAN, J. Native Americans as teacher trainers: Anatomy and outcomes of a cultural immersion project. Journal of Equity and Leadership, 2(2), 100-109, 1982.

MARGOLIS, J. Improving relationships between mentor teachers and student teachers: engaging in a pedagogy of explicitness. The New Educator, 3, 75-94, 2007. 
Repensando as conexões entre a formação na universidade e as experiências de campo na formação de professores em faculdades e universidades

METCALF, K. \& KAHLICH, P. Laboratory experiences ad transition from campus to field. In D.J. Mclntyre \& D. M. Byrd (Eds.) Preparing tomorrow's teachers: The field experience. p. 97-114, 1996.

MOJE, E.; CIECHANOWSKI, K.' KRAMER, K.; ELLIS, L.; CARRILLO, R.; COLLAZO, T. Working toward third space in content area literacy: an examination of everyday funds of knowledge and discourse. Reading Research Quarterly 39(1), 38-70, 2004.

PATTERSON, R.S.; MICHELLI, N.M. \& PACHECO, A. Centers of pedagogy. San Francisco: Jossey-Bass, 1999.

POINTER MACE, D. Teacher practice online: sharing wisdom, opening doors. New York: Teachers College Press, 2009.

POST, L.; PUGACH, M.; HARRIS, S.; HUGHES, M. The Teachers-In-Residence Program: Veteran urban teachers as teacher leaders in boundary-spanning roles. In K. Howey, \& N. Zimpher (2006) (Eds). Boundary spanners: a key to success in urban P-16 university-school partnerships. p. 211-236. Washington, DC: American Association of State Colleges and Universities and Land Grant Colleges, 2006.

RENNERT-ARIEV, P. The hidden curriculum of performance-based teacher education. Teachers College Record, 110(1), 105-138, 2008.

ROSAEN, C. \& FLORIO-RUANE, S. The metaphors by which we teach: Experience, metaphor and culture in teacher education. In M. COCHRAN-SMITH, S. NEMSER \& D.J. MCINTYRE (Eds). Handbook of research on teacher education. $3^{\text {rd }}$ edition, p. 706-731. New York: Routledge, 2008.

SAWCHUCK, NCATE offers multiple reaccreditation paths. Education Week, 28(36), p. 11, 2009.

SEIDEL, B. \& FRIEND, G. Leaving authority at the door: equal status, communitybased experiences and the preparation of teachers for diverse classrooms. Teaching and Teacher Education, 18, 421-433, 2002.

SLEETER, C. Preparing white teachers for diverse students. In M. COCHRANSMITH, S. FEIMAN-NEMSER \& D. J. MCINTYRE (Eds). Handbook of research on teacher education $3^{\text {rd }}$ edition. p. 559-582. New York: Routledge, 2008a.

SLEETER, C. Equity, democracy, and neo-liberal assaults on teacher education. Teaching and Teacher Education, 24(8), 1947-1957, 2008b.

SMAGORINSKY, P.; COOK, L. S. \& JOHNSON, T. S. The twisting path of concept development in learning to teach. Teachers College Record, 105(8), 13991436, 2003.

SOJA, E. Thirdspace. Malden, MA: Blackwell, 1996. 
STODDART, T. \& FLODEN, R. Traditional and alternative routes to teacher certification: Issues, assumptions and misconceptions. In K. ZEICHNER, S. MELNICK \& M. L. GOMEZ (Eds.). Currents of reform in pre-service teacher education. p. 80-108. New York: Teachers College Press.

STONES, E. \& MORRIS, S. Teaching practice: Problems and perspectives. London: Methuen, 1977.

TATTO, T. Examining the values and beliefs about teaching diverse students: Understanding the challenges for teacher education. Educational Evaluation and Policy Analysis, 18(2), 155-180, 1996.

TOM, A. Redesigning teacher education. Albany, SUNY Press, 1997.

TROEN, V.; KAMII, M.; \& BOLES, K. From carriers of culture to agents of change: teacher-initiated professional development in the learning/teaching collaborative inquiry seminars. Paper presented at the annual meeting of the American Educational Research Association, Chicago, 1997.

TUOMI-GROHN, T. Developmental transfer as a goal of internship in practical nursing. In T. TUOMI-GROHN \& Y. ENGESTROM (Eds). Between school and work: new perspectives on transfer and boundary- crossing. p. 199-232. Bingley, UK: JAI Press, 2007.

TURNEY, C.; ELTIS, K. TOWLER, J. \& WRIGHT, R. A new basis for teacher education: The practicum curriculum. Sydney: University of Sydney Press, 1985.

VALENCIA, S.; MARTIN, S.; PLACE, N. \& GROSSMAN, P. (in press). Complex interactions in student teaching: Lost opportunities for learning. Journal of Teacher Education.

VICK, M. It's a difficult matter: historical perspectives on the enduring problem of the practicum in teacher preparation. Asia-Pacific Journal of Teacher Education, 34(2), 181-198, 2006.

WISE, A. Legislated learning: the bureaucratization of the American classroom. Berkeley: University of California Press, 1979.

ZEICHNER, K. Beyond the divide of teacher research and academic research. Teachers \& Teaching, 1(2), 153-172, 1995.

ZEICHNER, K. hDesigning educative practicum experiences for prospective teachers. In K. ZEICHNER, S. MELNICK, \& M.L. GOMEZ (Eds). Currents of reform in pre-service teacher education. p. 215-234. New York: Teachers College Press, 1996.

ZEICHNER, K. Beyond traditional structures of student teaching. Teacher Education Quarterly, 59-64, 2002. 
Repensando as conexões entre a formação na universidade e as experiências de campo na formação de professores em faculdades e universidades

ZEICHNER, K. Becoming a teacher educator. Teaching and Teacher Education, 21, 117-124, 2005.

Zeichner, K. Professional development schools in a culture of evidence and accountability. School-University Partnerships, 1(1), 9-17, 2007.

ZEICHNER, K. Neo-liberal ideas and the transformation of teacher education in the U.S. Keynote address presented at the International Conference, Justice, Equality and Efficiency: Educational Policy Under the Situation of Multiple Societies. East China Normal University Shanghai, China, 2008.

ZEICHNER, K. \& CONKLIN, H. Teacher education programs. In M. CochranSmith \& K. Zeichner (Eds). Studying teacher education. p. 645-735. New York; Routledge, 2005.

ZEICHNER, K. \& MELNICK, S. The role of community field experiences in preparing teachers for cultural diversity. In K. Zeichner, S. Melnick \& M.L. Gomez (Eds). Currents of reform in pre-service teacher education. p. 176-198. New York: Teachers College Press, 1996.

ZEICHNER, K. \& MILLER, M. Learning to teach in professional development schools. In M. Levine \& R. Trachtman (Eds.) Making professional development schools work: Politics, practice and policy. p. 15-32. New York: Teachers College Press, 1997.

Notas

1 Isso inclui a atenção ao como a prática é ensinada em outros programas de preparação profissional (Grossman et al, 2009).

2 Cochran-Smith \& Lytle adotam o termo "terceira cultura" ao invés de terceiro espaço.

3 Tanto os sites de professores da Educação Básica quanto os sites dos formadores de professores podem ser acessados pelo seguinte link: www.insideteaching.org

${ }^{4}$ Por hiper racionalidade, entendo a extrema pressão nas instituições de formação de professores para racionalizar seus programas e sistemas de apoio ao estudante em vista de uma demanda por produtividade e flexibilidade que tem começado a interferir e minar a realização dos objetivos da formação de professores. Para uma discussão desses termos em relação à Educação Básica, ver Wise, 1979. Para uma discussão sobre medidas mais razoáveis e efetivas para desempenho na formação de professores, ver Zeichner (2008).

${ }^{5}$ Deve-se notar que a NCATE está, atualmente, engajada num grande esforço para discutir preocupações generalizadas sobre a hiper-racionalização em seu processo de acreditação.

\section{Correspondência}

Ken Zeichner - University of Washington-Seattle.

E-mail: kenzeich@uw.edu

Recebido em 24 de agosto de 2010

Aprovado em 23 de novembro de 2010 